\title{
Leishmaniosis Mimicking Oral Neoplasm in a Dog: An Unusual Manifestation of an Unusual Disease in Finland
}

\author{
By S. Saari ${ }^{1}$, J. Rasi ${ }^{2}$ and M. Anttila ${ }^{1}$ \\ ${ }^{1}$ Department of Pathology and Field Extension, National Veterinary and Food Research Institute, Helsinki, and \\ ${ }^{2}$ Small Animal Clinic of Turku City, Finland.
}

Canine leishmaniosis is a systemic protozoal disease, which is caused by a haemoflagellate of the genus Leishmania and transmitted by sandflies (Diptera: Phlebotomus spp.). The prevalence of the disease depends on the presence of insect vectors, and consequently the disease is endemic only in the Mediterranean area in Europe. Clinically the most important species in Europe is Leishmania infantum (Slappendel \& Ferrer 1998). It is an important pathogen in humans as well as in dogs. In Finland only sporadic cases of canine leishmaniosis have been reported in imported dogs (Saari et al. 1992) as in the other Nordic countries, Sweden (Johansson et al. 1998), Norway (Tharaldsen \& Vollset 1996) and Denmark (Kristensen et al. 1995). A long incubation period varying from a few weeks to 5 years is typical for the disease. Clinical symptoms include chronic wasting, somnolence, anorexia, undulating fever, diarrhoea, lymphadenopathy, arthritis, and dermatitis (Slappendel \& Ferrer 1998). The involvement of the mucous membranes is very rare in canine leishmaniosis (Font et al. 1996). In this report we describe a case of canine leishmaniosis that presented clinically as an oral nodular tumor, which was examined histologi- cally due to a suspicion of a malignant oral neoplasia.

The 7-year-old male mongrel dog had had a persistent anaemia for about a year with clinical signs and laboratory findings suggestive of immunohaemolytic anaemia. The dog had undergone a splenectomy and had been treated with corticosteroids for 9 months. The dog was presented to the clinician with a $4 \times 2 \times 1 \mathrm{~cm}$ protruding nodule on the ventral surface of the tongue. The tumor was surgically excised, fixed in $10 \%$ buffered formalin and sent for histopathological examination to the Department of Pathology and Field Extension, National Veterinary and Food Research Institute, Helsinki, Finland.

The sample was processed routinely and stained with haematoxylin and eosin (HE) and acid orcein giemsa (AOG). Histologically there was a fairly well demarcated inflammatory cell infiltrate elevating the overlying epithelium and expanding the fibrous stroma that was mainly composed of foamy macrophages with lesser number of plasma cells and fewer lymphocytes. The macrophages contained numerous intracytoplasmic round to oval organisms $(2-3 \mu \mathrm{m}$ in diameter) each with a single basophilic nucleus 


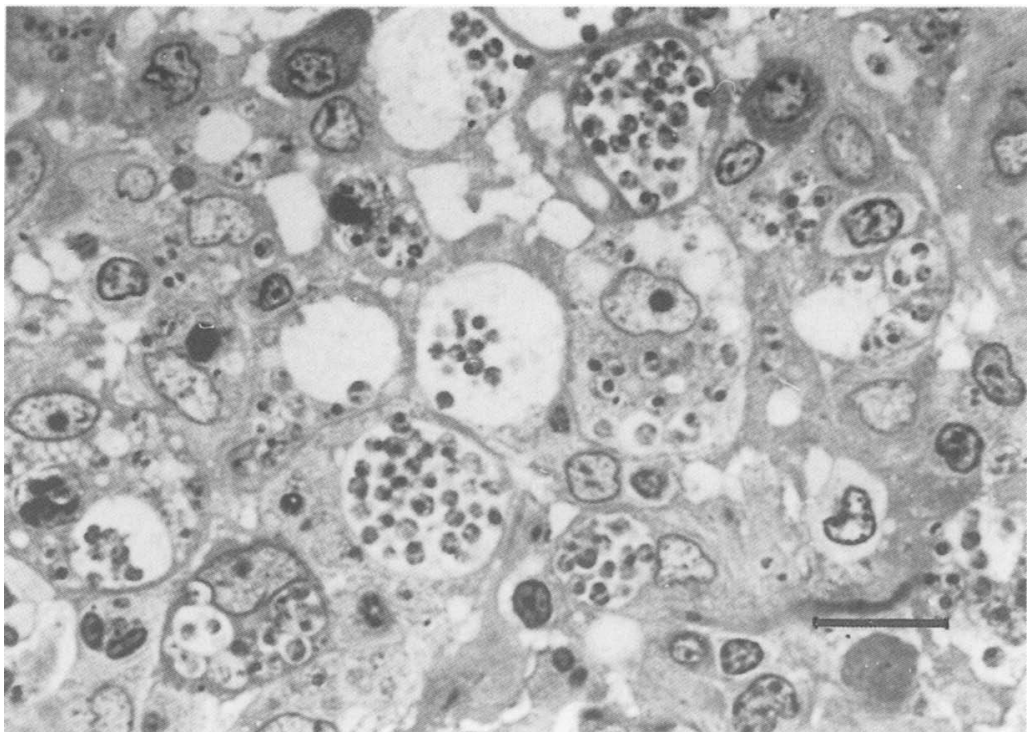

Figure 1. Nodular tumour like lesion from the mouth of a 7-year-old male mongrel dog composed of macrophages with a myriad of intracytoplasmic protozoal organisms. Plasma cells and lymphocytes are also present. Toluidine blue -stained semithin section. Bar $=10 \mu \mathrm{m}$.

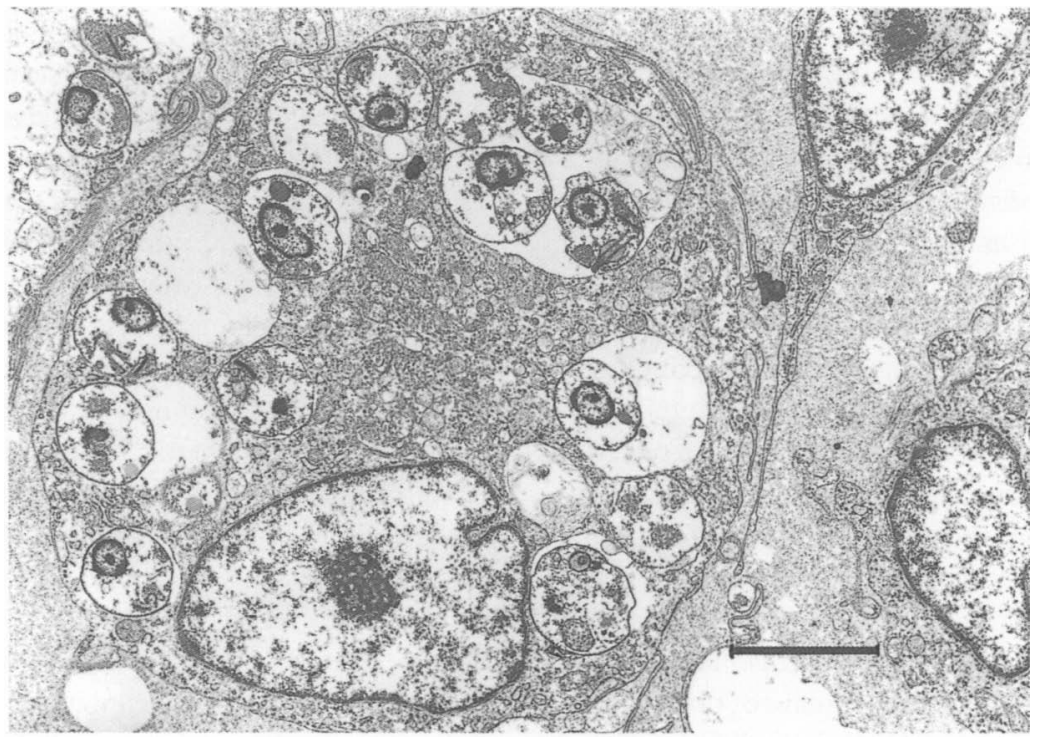

Figure 2. Transmission electron micrograph of a macrophage with clearly visible amastigotes within the cytoplasm. Bar $=3 \mu \mathrm{m}$. 


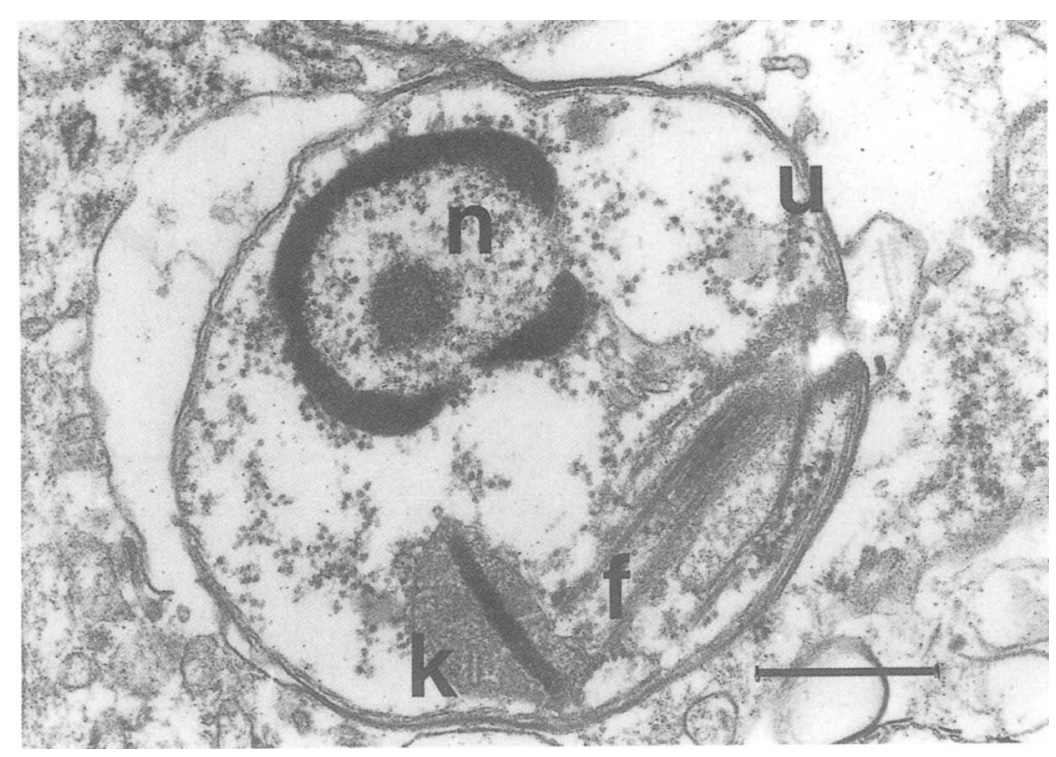

Figure 3. Transmission electron micrograph with typical morphological features of the amastigote: $n=n u-$ cleus, $\mathrm{f}=$ flagellum, $\mathrm{k}=$ kinetoplast, $\mathrm{u}=$ unit plasma membrane. $\mathrm{Bar}=0.5 \mu \mathrm{m}$.

and a barely discernable kinetoplast. The organisms stained positively with AOG.

The histological findings were highly suggestive of leishmaniosis. Semithin $(1 \mu \mathrm{m})$ sections for light microscopy and ultrathin sections for transmission electron microscopy (TEM) were used to confirm the diagnosis, since they allowed better visualisation of the morphology of the organisms. The formalin fixed samples were postfixed in $1 \%$ osmium tetroxide, dehydrated in ethanol, and embedded in epoxy resin. Semithin sections were stained with $1 \%$ toluidine blue and examined with light microscope. Ultrathin sections were cut from those blocks in which organisms were seen after toluidine blue staining (Fig. 1.) and stained with uranylacetate and lead. They were examined with JEM $100 \mathrm{~S}$ transmission electron microscope (Jeol, Tokyo, Japan). Numerous non-flagellate forms, amastigotes were present in the dilated phagolysosomes of macrophages (Fig. 2). Typical fea- tures of the amastigotes of Leishmania spp., a cytoplasmic kinetoplast and a short non-emerging flagellum, were present. Morphological features of the amastigote, often referred as Leishman-Donovan (L-D) body, are summarised in Fig. 3.

Treatment of the dog with sodium stibogluconate $\left(\right.$ Pentostam ${ }^{\circledR}$, Glaxo Wellcome, United Kingdom) (40 mg/kg/day/s.c/ 30 days) was started immediately after the diagnosis. The dog suffered from adverse drug reactions during the treatment and was vomiting several times a day. About 2 months later the dog developed a necrotizing dermatitis on the both ear pinnae. The owners decided to euthanize the animal, but did not want necropsy to be performed.

This case represents an unusual manifestation of leishmaniosis in a dog. The owners had failed to inform the clinician of the Mediterranean origin of the dog. Detailed history was ob- 
tained after the diagnosis, and revealed that the dog was born in Spain. It was brought to Finland at the age of 1 year. It appears that in this case the incubation period was very long, since the dog had lived in Finland over 5 years without showing any obvious clinical signs. However, the dog had visited Spain for a week a couple of months before the onset of anaemia, so it is possible that it was infected during that time period. Canine leishmaniosis is usually a systemic disease, thus it is likely that in this case the oral tumour-like lesion was a manifestation of the systemic disease, even if the oral and other mucocutaneous lesions as well as tumour-like presentations are considered very rare in canine leishmaniosis (Font et al. 1996). Although there are several methods to diagnose canine leishmaniosis, the mucosal manifestation is such a rare form in dogs that histopathology or cytology is required for a correct diagnosis.

Nodular or ulcerating mucosal lesions are sometimes present in human patients with visceral leishmaniosis, especially in Sudan and elsewhere in East Africa, and India (Oster \& Chulay 1991). This case shows that leishmaniosis can appear with variable and non-specific clinical signs. The incubation period that can last several years may cause diagnostic problems especially when dogs have been imported from endemic areas to countries where leishmaniosis does not naturally occur. Although sandflies are the natural vectors of this zoono- sis, the transmission of leishmaniosis by direct contact between infected material and either mucous membranes or broken skin has been reported (Slappendel \& Ferrer 1998, Harris 1994). Thus certain caution is necessary when handling an infected dog or material from it.

\section{References}

Font A, Roura X, Fondevila D, Closa JM, Mascort J, Ferrer L: Canine mucosal leishmaniasis. J. Am. Anim. Hosp. Assoc. 1996, 32, 131-137.

Harris MP: Suspected transmission of leishmaniasis. Vet. Rec. 1994, 135, 339.

Johansson M, Frendin J, Ferrer L, Lindberg $R$ : Leishmanios hos hund i Sverige (Leishmaniasis in dogs in Sweden) Sv. Vet. Tidn. 1998, 50, 293297.

Kristensen F, Jensen AL, Falmer-Hansen J: Leishmaniasis hos hund - en relevant sygdom i Danmark (Leishmaniasis in dogs: a disease of relevance in Denmark). Dansk Veterinaertidsskrift 1995, 78, 913-916.

Oster CN, Chulay JD: Visceral leishmaniasis (Kalaazar). In: Strickland GT (ed.): Hunter's Tropical medicine (7. ed.). W. B. Saunders Company, Philadelphia, PA, USA, 1991, pp. 642-648.

Saari S, Nikander S, Eriksson J, Sarjamo P: Viskeraalinen leishmanioosi koiralla - terveysriski myös ihmiselle (Visceral leishmaniosis in dogs A human health hazard). Suom. Eläinlääkäril. 1992, 98, 244-249.

Slappendel RJ, Ferrer L: Leishmaniasis. In: Greene CE (ed.): Infectious diseases of the dog and cat (2. ed). W.B. Saunders Company, Philadelphia, PA, USA, 1992, pp. 450-458.

Tharaldsen J, Vollset I: Eksotiske parasitter hos hund og katt (Exotic parasites of dogs and cats). Norsk Vet. Tidsskr. 1996, 108, 761-770.

(Received February 18, 2000; accepted February 22, 2000).

Reprints may be obtained from: S. Saari, National Veterinary and Food Research Institute, P.O. Box 368, FIN00231 Helsinki, Finland. E-mail: seppo.saari@eela.fi, tel: +358-9-3931894, fax: +358-9-3931960. 